\title{
FUNCIONES Y ESTRATEGIAS FUNDAMENTALES DE LA GUÍA-TUTELA ENTRE IGUALES EN LAS PRIMERAS EDADES'
}

\author{
Vargas Cano, J.D. y Villares Bermejo, L. \\ Universitat Rovira i Virgili
}

\begin{abstract}
RESUMEN. En este trabajo presentamos un marco teórico sobre la guía-tutela entre iguales en las primeras edades. Desde una perspectiva pluridimensional describimos sus principales características, en contraste con la guía tutela adulto-niño/a. Detallamos los principales patrones de organización de la guía-tutela y, finalmente, concretamos las características de una tutoría eficaz en estas edades.
\end{abstract}

PALABRAS CLAVE: perspectiva pluridimensional, interacción entre iguales, guíatutela, desarrollo temprano.

ABSTRACT. In this work we displayed a theoretical frame on the peer guide-tutoring in the early ages. From a pluridimensional perspective we described its main characteristics, in contrast to the adult-child guide. We detailed the main patterns of organization of the guide and, finally, we made specific the characteristics of an effective position of a tutoring in these ages.

KEY WORDS: pluridimensional perspective, peer interation, guide, early development.

\section{Introducción}

En el marco de la nueva propuesta del sistema educativo (LOGSE) se propugna la interacción entre iguales en los primeros niveles de la educación infantil como una estrategia y orientación psicopedagógica que guía el currículum de esta etapa.

A pesar de este interés mostrado desde las instancias normativas de la educación, todavía son escasas (en nuestro contexto) las investigaciones que abordan desde una perspectiva estrictamente psicopedagógica cuáles pueden ser los mecanismos y resultados cognitivos y educativos a que puede conducir la interacción entre iguales en estas primeras edades (0;0-3;0 años).

En este trabajo, y desde una perspectiva teórica pluridimensional (Beaudichon, Verba \& Winnykamen, 1988) ofrecemos una reflexión en torno al tópico de la guía-

1. Este trabajo ha sido posible, en parte, gracias a una beca de Formació d'Investigadors financiada por la Generalitat de Catalunya (FI-PG/96-7.010). 
tutela entre iguales en las primeras edades. Para ello ofrecemos un plano de aproximación teórico fundamentado en una aproximación pluridimensional a los conceptos de modalidades sociocognitivas de construcción del saber, y las especificidades del desarrollo de la guía-tutela.

\section{La perspectiva pluridimensional en el estudio de la interacción entre iguales: más allá de Piaget y Vygotsky.}

El soporte teórico de nuestra propuesta acerca de la guía-tutela y la interacción entre iguales no se define a partir de la adopción de una de las dos clásicas perspectivas en el área: la piagetiana o la vygotskyana, sino que pretendemos trascender una falsa dicotomía entre ambos enfoques para integrar sus aspectos complementarios en una nueva dimensión, siendo conscientes de las dificultades epistemológicas que pueden derivarse de tal empeño; dado que, grosso modo, Piaget representa la perspectiva del constructivismo individual y Vygotsky la de del constructivismo social, por lo que resulta complejo el intento de integración de ambas visiones en una plataforma teórica que permita el abordaje de las interrelaciones entre interacción niño/a-niño/a y desarrollo cognitivo.

Actualmente, en un intento de integrar las aportaciones de ambas perspectivas surge la perspectiva pluridimensional (Beaudichon, Verba \& Winnykamen, 1988) que investiga díadas simétricas y asimétricas. La perspectiva pluridimensional posibilita una aproximación a la comprensión de la aportación de lo individual y lo social en el desarrollo cognitivo, puesto que observa ambos enfoques microgenéticamente, en sus procesos y explicaciones (Sastre y Pastor,en prensa; Pastor y Sastre, 1994).

Dentro de este enfoque se encuadran una serie de investigadores inmersos en el marco teórico piagetiano clásico (en el sentido que conservan y defienden el papel de autorregulación del individuo) pero que integran (filtrando a través de un matiz piagetiano) algunas aportaciones del constructivismo sociocultural vygotskiano: se interesan por el efecto de la interacción en la construcción del conocimiento, que desde la perspectiva pluridimensional es bidireccional; los dos (o más) sujetos integrantes de la situación de interacción son protagonistas activos.

Destacamos en este sentido las investigaciones de Beaudichon, J., Verba, M. \& Winnykamen, F. (1988); Pastor, E. y Sastre, S. (1994); Sastre, S. y Pastor, E. (1997); Stambak, M. \& Sinclair, H. (1993); Verba, M. (1993); Verba, M. \& Winnykamen, F. (1992); Verba, M. (1994), y especialmente las hipótesis más generales de la perspectiva pluridimensional, que pueden resumirse como sigue:

- Afirmar que no existe una única modalidad de construcción de saber en la interacción social. En estas situaciones se dan diferentes modalidades, que son correspondientes a una cierta pluralidad de conocimientos (Verba, M. \& Winnykamen, F., 1992; Verba, M., 1994; Beaudichon, J., Verba, M. \& Winnykamen, F. 1988). Se supone que existen diversidad de mecanismos de construcción de saberes (organización cognitiva) (Roux, J. P. \& Gilly, M, (1993); Fraysse, J.C. (1992); Gilly, M. (1980); Gilly, M. (1988; 1989; 1989b) en función, básicamente, de si la situación de la interacción es simétrica o asimétrica. 
- Los mecanismos que se postulan para explicar la posibilidad de progreso cognitivo en situaciones de interacción social son, entre otros, (Verba, 1994; Beaudichon, Verba \& Winnykamen, 1988) la imitación, la co-construcción (conflicto y cooperación), y la transmisión de conocimientos-adquisición (guía-tutela).

Vamos a comentar con más detalles estas diferentes modalidades sociocognitivas de construcción de saber para poder situar con más precisión el papel de la guía-tutela entre iguales dentro de este cuadro de modalidades de organización de la interacción.

\section{Modalidades sociocognitivas de construcción de saber}

Antes de comenzar a comentar teóricamente las principales características de la guía-tutela en las primeras edades es necesario que la contextualicemos adecuadamente. Desde la perspectiva pluridimensional podemos contemplar, en general, tres modos de co-elaboración en los intercambios sociales entre niños/as de corta edad (Verba \& Isambert, 1987; Beaudichon, Verba \& Winnykamen, 1988; Verba el al., 1982; Verba, 1994; Verba, 1997), siendo la guía-tutela uno de ellos. Vamos a comentar brevemente estos modos de co-elaboración:

1. El modo de imitación: en este caso la actividad de un niño/a desencadena la actividad en otro niño/a y le sirve de modelo; el segundo niño/a entonces produce la actividad observada (reproducción) con alguna modificación, estableciendo relaciones medios-fines a partir de la actividad observada o yendo más allá de las metas del modelo por extensión de la actividad observada.

2. Modo de co-construcción: en esta modalidad de interacción los niños/as utilizan sus ideas y acciones juntos para lograr un fin compartido que puede ser predefinido o desarrollado durante el curso de la interacción. La actividad cognitiva es inmediatamente relacionada y llevada a cabo mediante idénticas acciones (por ejemplo, cada niño/a coloca un bloque para construir una torre), o mediante diferentes acciones que complementan a las del otro/a. En cada momento de la actividad el proceso de interacción a menudo está organizado por la negociación, con el fin de llegar a establecer una comprensión mutua y un acuerdo entre compañeros/as. En la modalidad de co-construcción el control de las actividad (o gestión) es compartido por ambos integrantes.

En general, la co-construcción puede adoptar dos manifestaciones diferentes: coconstrucción consensuada o co-construcción conflictual.

3. Actividad guiada: la actividad de uno de los niños/as es dirigida a través de la ayuda del compañero/a. La relación social entre los niños/as posibilita la acción a través de la facilitación, la demostración, o el soporte socio-emocional de la guía del compañero/a. La dirección está controlada por el compañero-guía, que evalúa la intención o el producto con relación a una meta explícita o inferida.

Los tres modelos básicos de funcionamiento interactivo descrito (imitación, coconstrucción y guía-tutela) conforman un continuo desde el polo individual al social y desde el social al individual en la elaboración cognitiva de los niños/as. En la imitación la centración está más cerrada en el polo individual, donde el sujeto controla y desarrolla su plan de acción y posee un considerable margen para transformarlo. En la actividad guiada el niño/a a menudo depende del "input" del compañero/a más 
habilidoso, el cual controla la actividad de acuerdo al plan que tiene en mente o construye a través de la inferencia. La co-construcción está situada entre estos dos modos de organización, donde las metas y el control cognitivo de la actividad son compartidos por los dos compañeros/as (Beaudichon, Verba \& Winnykamen, 1988).

Una vez delimitado en qué contexto se sitúa la guía-tutela dentro de las diferentes posibilidades de organización de la interacción entre iguales, podemos pasar a comentar con más extensión sus características y especificidades en las primeras edades.

\section{Guía-tutela en las primeras edades}

La actividad guiada entre iguales en las primeras edades está fundada en procesos de transmisión-apropiación, de manera que una idea que es presentada en un momento dado, puede ser traspasada al compañero/a mediante medios informativos y justificativos. El niño/a que tiene la idea también orquesta la interacción. Él o ella actúa más o menos como un guía o tutor en el desarrollo de la idea mientras también intenta tener en cuenta el estado mental del compañero/a y sus reacciones. Las cuestiones y posibles objeciones del compañero/a provocan clarificación y explicaciones complementarias.

Se han definido varias manifestaciones diferentes de esta modalidad en la interacción entre iguales (Verba, 1994): a) la propuesta que desencadena la secuencia de guía-tutela no está conectada con la propuesta previa (o la actividad previa del compañero/a) o b) la propuesta que desencadena la secuencia está relacionada con una previa. En este caso, el niño/a que está gestionando la interacción acepta la idea del otro niño/a e incluso la incorpora en el seno de una construcción más comprensiva o rectifica o modifica la propuesta de trabajo del compañero/a y la extiende.

Por otra parte, es posible considerar que la guía puede ser apropiada para llevar a cabo la tarea. No obstante, en algunos casos la guía puede ser inapropiada si el compañero/a-tutor proporciona información errónea al compañero/a. Esta forma puede ser entendida como "guía-tutela inapropiada" (Verba, 1994).

En esto momento de la exposición podemos preguntarnos si la guía-tutela entre iguales es similar a la guía-tutela entre adulto-niño/a. Trataremos de reflexionar en torno a este tópico en el siguiente apartado.

\subsection{Guía-tutela entre iguales y entre adulto niño}

La pregunta con la que finalizábamos el apartado anterior no tiene una respuesta excluyente. En otras palabras, la guía-tutela entre niños/as presenta ciertas similitudes con la guía-tutela en la interacción adulto-niño/a, pero difiere en otros aspectos. Azmitia (1988) muestra que la guía en una interacción entre iguales experto-novato tiene efectos positivos en una tarea de resolución de problemas. Ellis y Rogoff (1982; 1986) analizaron la tutoría como un problema para ser resuelto en sí mismo, donde tres dimensiones (gestión de la tarea, gestión de la información y gestión de la comunicación) sobretasaban las capacidades del niño/a para la gestión simultánea. Aunque el primer y el tercer componente son bastante bien manejados, el componente de 
información es difícilmente controlado en las interacciónes entre iguales en las primeras edades.

Diversas investigaciones sugieren que los niños/as pequeños/as poseen conocimiento acerca del rol y los procedimientos implicados en la tutoría en la edad escolar (Cooper, Marquis, \& Edward, 1986; Verba \& Winnykamen, 1992), y que se comienzan a vislumbrar signos del comienzo de las conductas de tutoría entre los 3;04;0 años (Verba, 1994; Verba \& Marcos, 1995).

Por otra parte algunas de las investigaciones sobre la interacción entre iguales (Cooper et al., 1986; Wertsch \& Hickmann, 1987; Wood, Bruner, \& Ross, 1976; Verba \& Winnykamen, 1992; Verba, 1994; 1998) sugieren que los niños/as enseñan de forma diferente al adulto. En efecto, parece que los iguales tutores están más concentrados en las demandas inmediatas de la tarea y que utilizan más demostraciones y modelamientos directos. Además, proporcionan asistencia abundante, llegando incluso a desarrollar la tarea ellos/as mismos (Ellis y Rogoff, 1982; 1986) en lugar de dejársela desarrollar al novato/a. El tutor/a adulto, por su parte, proporciona más directrices, utiliza un canal verbal de instrucciones más complejo y, en general, sustituye la información de estructura más compleja por otra de más fácil comprensión (Shute, Foot \& Morgan, 1992). En este mismo sentido, Cazden (1988) observó que los niños/as tutores de menor edad produjeron más acciones directivas, realizaron más demostraciones y ofrecieron menos explicaciones que los tutores mayores. Otras investigaciones (Cooper, Marquis \& Edward, 1986) compararon diferentes grupos de edad (5;0-9;0 años y $7 ; 0-12 ; 0$ ) y encontraron que los tutores mayores ofrecieron más explicaciones y realizaron más planificaciones que los jóvenes.

La gestión cognitiva de la tarea instruccional es más sencilla en la interacción adulto-niño/a dado que las competencias/estrategias generales del adulto compensan las posibles lagunas en conocimientos relacionados con la tarea. En las interacciones entre niños/as con un experto y un novato, no obstante, la asimetría es temporal de manera que la relación de conocimiento entre los compañeros/as se dirige hacia la simetría conforme el novato/a aprende.

Uno de los principales inconvenientes con los que puede encontrarse un tutor/a en estas primeras edades es el desconocimiento más o menos importante de la actividad que debe "enseñar" a su compañero/a. La consecuencia de una insuficiente maestría sobre la tarea/actividad radica en que puede inducir al niño/a experto a probar su conocimiento al mismo tiempo que el novato/a está siendo asistido en su proceso de construcción del saber relacionado con la tarea/actividad. A diferencia del adulto, el niño/a tutor reconcilia ambas actividades: aprender y procurar que el compañero/a aprenda. Este proceso permite que guiando al compañero/a el niño/a tutor pueda tener la oportunidad de "comprobar" su nuevo y recientemente adquirido saber y/o consolidarlo más a través de la práctica con el material (Verba; 1998).

La importancia de todo lo comentado hasta el momento radica en que se sugiere que las habilidades para proporcionar explicaciones y planificar se desarrollan con la edad. En efecto, los resultados de las investigaciones comentadas sugieren que los niños/as más pequeños se concentran más en la ejecución de la tarea y permanecen más ligados a la acción, mientras que los niños/as mayores realizan mayor número de ayudas instrumentales y verbales. 
Una vez ya hemos comentado cuáles son las principales diferencias y semejanzas entre la guía-tutela adulto-niño/a y la guía-tutela entre iguales, podemos pasar a comentar en qué estructuras puede organizarse la guía-tutela en las primeras edades.

\subsection{Patrones específicos de guía-tutela en la interacción entre iguales}

Desde la perspectiva pluridimensional en el estudio de los procesos de interacción social se intenta matizar y concretar en la mayor medida posible las diferentes formas de organización que pueden adoptar las modalidades sociocognitivas de construcción de saber. Fruto de este esfuerzo, recientemente se han establecido patrones específicos de guía-tutela en la interacción entre iguales (Verba, 1998). Los patrones sugeridos son los siguientes:

- Instrucción del experto/a. El experto/a desarrolla una iniciativa, proporcionando diferentes tipos de información al novato/a y actúa por él/ella. Si el novato/a está pasivo el tutor/a no intenta su implicación; o si el novato/a quiere actuar el experto/a no le deja hacerlo.

- Gestión compartida. Surge como iniciativa del experto/a o del novato/a. En este caso el experto/a informa y facilita, y el novato/a le sigue, actuando según las propuestas o realizando demandas. El experto sólo ejecuta la acción cuando el novato/a falla.

- Gestión del novato/a con soporte del experto/a. El novato/a desarrolla una actividad, y el experto/a la evalúa en función de las necesidades detectadas en el compañero/a. En estas situaciones, el experto/a sólo proporciona una mínima asistencia (facilitación).

- Gestión del novato/a con control del experto/a. El novato/a encuentra la solución del problema sin ayuda del tutor/a, quien simplemente observa y realiza evaluaciones esporádicas.

- Gestión del novato/a. El novato/a corre con toda la responsabilidad de la actividad, incluso cuando necesita ayuda. Se da en casos en que el tutor/a no muestra un interés elevado por la actividad.

Éstas son algunas de las formas en qué podría organizarse la interacción de guíatutela entre iguales en las primeras edades. Otra cuestión interesante al respecto sería señalar cuáles podrían ser las características de la tutoría eficaz, dado que este aspecto puede orientar la intervención psicopedagógica con los sujetos de estas edades iniciales, con el objetivo de optimizar al máximo los posibles resultados cognitivos y educativos derivados de la guía-tutela entre iguales en este momento del desarrollo.

\subsection{Funciones y características de la tutoría eficaz.}

En la interacción entre iguales resulta interesante destacar cuáles podrían ser las características de un tutor eficaz. (Wood, Bruner et Ross, 1976) sugieren que la interacción social y cognitiva se realiza mediante procesos de "andamiaje" que suponen, para el tutor/a, la consecución de la implicación de los dos compañeros/as en la tarea, la simplificación de la misma (dejando al "novato/a" ejecutar las subrutinas que puede realizar), la ayuda a la motivación, el ofrecimiento de críticas, el control de las frus- 
traciones, las demostraciones y la presencia de una teoría implícita de los actos necesarios para realizar la tarea y de una teoría de las características del modo de actuación del novato/a.

Tradicionalmente se ha entendido que la capacidad de tutorar a otro/a requería, en general, una representación de la función de guía, estrategias apropiadas de tutela, sensibilidad hacia las necesidades del aprendiz y cierta conciencia acerca de las demandas de la tarea. Algunos estudios (Ellis y Rogoff, 1982) han sugerido que desde los 7;0 años los niños/as poseen ya una buena representación de la función tutorial: un maestro/a, entienden ellos/as, debe mostrar responsabilidad y autoridad, ayudar al alumno/a y proporcionarle conocimiento.

Ahondando en las capacidades de los niños/as más pequeños/as, diversos investigadores han realizado observaciones de carácter semi-natural en centros de educación infantil, observando que entre las edades de 1;6 años y 2;0 años los niños/as muestran gran interés en la actividad de los iguales, y son sensibles a las dificultades que encuentran los otros/as (Verba, Stambak \& Sinclair, 1982; Verba \& Winnykammen, 1992; Verba, 1994; 1998). De esta forma, es posible observar, en actividades espontáneas centradas en los objetos, conductas esporádicas de ayuda cuando un niño/a que "sabe" (experto) ha desarrollado una actividad exitosamente e infiere una necesidad en el compañero/a para completar la misma actividad Este tipo de asistencia consiste en facilitar la acción del compañero/a proporcionándole el objeto apropiado, apartando obstáculos, etc. Estas conductas de ayuda a menudo aparecen acompañadas de manifestaciones pro-sociales. En este sentido, las interacciones de este tipo pueden ser consideradas como precursoras de la tutoría, en el sentido que un niño/a ayuda al otro/a a superar un obstáculo o completar una nueva actividad. A la edad de 3;0-4;0 años, con la adquisición del lenguaje, la tutoría aparece en una estructura más organizada, en algunos episodios de actividad compartida cognitiva. El tutor/a entonces ofrece información y directrices para la acción, evaluaciones y animaciones, extendiendo la actividad del tutorado mediante estrategias de modelación o realizando la tarea del compañero/a (Verba \& Isambert, 1987).

No obstante, en estas edades todavía existe una especial sensibilidad hacia la figura del adulto, de manera que entre la edad de 4;0-5;0 años diversas investigadoras han observado que las demandas son mucho más frecuentes con un compañero/a adulto que con un compañero/a igual (Verba \& Marcos, 1995).

Para conseguir una mejor comprensión del desarrollo de la tutoría, es necesario examinar las funciones específicas de estas acciones de enseñanza, tanto en su contenido como en su forma. A partir de los trabajos de Bruner y colaboradores (Wood, Bruner, \& Ross, 1976) y de las dinámicas que pueden observarse en las actividades compartidas (Verba, 1993b; 1994; 1998) tales funciones pueden ser definidas en relación con los fines mismos de la tutela (ver cuadro 1): Ilevar la comprensión del novato/a más allá del estado actual mediante la provisión de información verbal y no verbal y mediante feedback, sosteniendo la actividad cognitiva mediante ayudas que faciliten la tarea, manteniendo al tutelado motivado por medio de la animación, y manteniendo al mismo tiempo la cooperación gracias al establecimiento de una relación social y afectiva positiva. Como contrapartida, el novato/a debe ser consciente de sus limitaciones y solicitar/demandas ayuda. Parece que existe un proceso cons- 


\section{Participación del guía.}

FUNCIÓN DE COMPREHENSIÓN (Ayuda instrumental).

1. Informar y proporcionar direcciones (establecer finalidades, dar instrucciones, explicar, señalando detalles importantes), respondiendo las preguntas del novato.

2. Demostrar/modelar y dejar actuar al otro niño/a.

\section{FUNCIÓN DE FEEDBACK}

3. Ofrecer feedback (evaluar la acción actual o su resultado; corregir un error).

\section{FUNCIÓN DE FACILITACIÓN}

4. Proporcionar ayuda durante la ejecución: sosteniendo una pieza, señalado dónde estaba la pieza

5. Ayudar en la realización de la tarea: colocar una pieza en su sitio.

\section{FUNCIÓN DE IMPLICACIÓN EN LA ACTIVIDAD.}

6. Procurar atención: haciendo que el compañero/a actúe cuando no está atento o está pasivo.

\section{FUNCIÓN DE CONSTRUCCIÓN Y MANTENIMIENTO DE LA RELACIÓN SOCIAL}

7. Mantener la cooperación y establecer un lazo mediante el lenguaje o utilizando medios no verbales (miradas compartidas y sonrisas), manifestando alegría, proporcionando ánimos.

\section{Participación del novato/a.}

\section{DEMANDAS DE AYUDA}

8. Directas: demandas de información, evaluación o acción.

9. Indirectas: expresiones de dificultad, mirar al compañero/a....

Interacción simétrica (experto/a y novato/a)

10. Sustitución: el experto actúa por el novato y coloca la pieza en su lugar..

11. Obstáculo: el experto previene al novato de la actuación, mediante gestos o hablando.

12. Desacuerdo con el compañero/a, protestando, argumentando.

Ejecución de la tarea (experto/a o novato/a).

13. Ensayo y error.

14. Colocar la pieza en un lugar correcta o incorrectamente.

15. Autoevaluación: mirar el dibujo, haciendo comentarios respecto a la propia actividad. 
tructivo-evolutivo en este sentido, de manera que los niños/as pequeños comienzan a construir el dominio de la tutela haciendo la tarea comprensible, facilitando su ejecución y manteniendo la interacción, y luego, más tarde, adquieren los conocimientos necesarios para poder ajustar la instrucción a las necesidades del novato/a (Verba, 1998).

En relación con la especificación de la ayuda que un compañero/a puede proporcionar al otro/a es necesario hacer referencia a las categorías desarrolladas por investigadores como Wood et Middleton (1975) y Santolini et. al. (1996), y que resumimos brevemente como sigue:

1) Ayuda ofrecida por el guía-tutor durante la tarea en curso, previamente a la resolución de la misma: a) evocación de la tarea que se va a resolver; b) orientar la atención, permitir la implicación del compañero/a en el problema que se va a resolver; c) evocación de la finalidad del problema, del resultado que se pretende obtener; d) proporcionar los medios de resolución al compañero/a (se trata de informaciones parciales que permiten alcanzar el fin de la tarea); e) proporcionar un procedimiento (ofrecer el "cómo hacer").

2) Ayuda ofrecida después de la resolución de la tarea: a) evocación simple del resultado (el compañero/a comenta el resultado de la acción del otro/a, sin informarle si es correcto o no); b) corrección sin justificación: se trata de remarcar sin evaluar; c) corrección con justificación; d) ayuda a la autocorrección; e) felicitaciones.

Ellis y Rogoff (1986) sostienen que la condición esencial para proporcionar una asistencia efectiva al novato/a es la maestría en la tarea, de manera que un insuficiente conocimiento en el área o dominio que se intenta tutelar desemboca, generalmente, en una inefectiva guía-tutela. La asimetría de competencias entre compañeros/as depende del conocimiento específico de la tarea y de las competencias generales del tutor/a.

Además, la tutoría requiere de ambos compañeros/as el conocimiento de sus diferencias en estatus y rol. La maestría del niño/a en la tarea le confiere un estatus de conocedor y un rol implícito de enseñanza durante la interacción con el novato/a. Pero el novato/a también debe reconocer su estatus para aceptar las directrices del tutor/a, adoptando una función complementaria del que hace ("actor") y solicitando ayuda. En interacciones asimétricas estrictas, el estatus y la diferencia de rol están implícitas, pero cuando la asimetría es ligera o temporal, estas diferencias disminuyen y los estatus respectivos tienden hacia la simetría. Algunos autores (Forman \& Cazden, 1984; Verba \& Winnykamen, 1992) basándose en el conocimiento y las relaciones de poder entre compañeros/as afirman que la asimetría no es una entidad estable en la interacción entre iguales. En efecto, las relaciones interpersonales construidas a través de experiencias que son compartidas (Hartup, 1989; Bensalah, 1995) o establecidas en el curso de la interacción, pueden modificar el grado de asimetría. Incluso si la diferencia en estatus es explícita o implícitamente conocida, la competitividad natural de las relaciones entre niños/as tiende a orientar la asimetría de la situación novato/a-experto/a hacia un tipo más simétrico de interacción. 
En otras palabras, a partir de las investigaciones precedentes podemos sostener que cierto conocimiento básico sobre el repertorio de conductas de tutela ha sido adquirido ya antes de los 5;0 años.

\section{Conclusiones}

Desde una perspectiva teórica pluridimensional (Beaudichon, Verba \& Winnykamen, 1988) hemos ofrecido una reflexión en torno al tópico de la guía-tutela entre iguales en las primeras edades

Desde esta perspectiva pluridimensional sostenemos que no existe una única modalidad de construcción de saber en la interacción social. En estas situaciones se dan diferentes modalidades, que son correspondientes a una cierta pluralidad de conocimientos. Las modalidades que se postulan para explicar la posibilidad de progreso cognitivo en situaciones de interacción social son la imitación, la co-construcción (conflicto y cooperación), y la transmisión de conocimientos-adquisición (guíatutela).

Hemos visto que en la guía-tutela la actividad de uno de los niños/as es dirigida a través de la ayuda del compañero/a. La relación social entre los niños/as posibilita la acción a través de la facilitación, la demostración, o el soporte socio-emocional de la guía del compañero/a. La dirección está controlada por el compañero-guía, que evalúa la intención o el producto con relación a una meta explícita o inferida.

La guía-tutela entre niños/as presenta ciertas similitudes con la guía-tutela en la interacción adulto-niño/a, pero difiere en otros aspectos. Diversas investigaciones sobre la interacción entre iguales (Cooper et al., 1986; Wertsch \& Hickmann, 1987; Wood, Bruner, \& Ross, 1976; Verba \& Winnykamen, 1992; Verba, 1994; 1998) sugieren que los niños/as enseñan de forma diferente al adulto. Los iguales tutores están más concentrados en las demandas inmediatas de la tarea y utilizan más demostraciones y modelamientos directos. Los niños/as tutores de menor edad producen más acciones directivas, realizan más demostraciones y ofrecen menos explicaciones que los tutores/as mayores (Cazden, 1988). La gestión cognitiva de la tarea instruccional es más sencilla en la interacción adulto-niño/a dado que las competencias/estrategias generales del adulto compensan las posibles lagunas en conocimientos relacionados con la tarea. En las interacciones entre niños/as con un experto/a y un novato/a, no obstante, la asimetría es temporal de manera que la relación de conocimiento entre los compañeros/as tiende hacia la simetría.

Hemos sugerido que existe un proceso constructivo-evolutivo de las estrategias y procesos de la guía-tutela, de manera que los niños/as pequeños comienzan a construir el dominio de la tutela haciendo la tarea comprensible, facilitando su ejecución y manteniendo la interacción, y luego, más tarde, adquieren los conocimientos necesarios para poder ajustar la instrucción a las necesidades del novato/a (Verba, 1998). Diversas investigaciones sugieren que los niños/as pequeños/as poseen conocimiento acerca del rol y los procedimientos implicados en la tutoría en la edad escolar (Cooper, Marquis, \& Edward, 1986; Verba \& Winnykamen, 1992), y que se comienzan a vislumbrar signos del comienzo de las conductas de tutoría entre los 3;0-4;0 años (Verba, 1994; Verba \& Marcos, 1995). Ya antes, entre las edades de 1;6 años y 
2;0 años los niños/as muestran gran interés en la actividad de los iguales, y son sensibles a las dificultades que encuentran los otros/as (Verba, Stambak \& Sinclair, 1982; Verba \& Winnykammen, 1992; Verba, 1994; 1998), de manera que son capaces de ayudarles en la resolución de la tarea.

\section{Referencias Bibliográficas}

AZMITIA, M. (1988): Peer interaction and problem solving: When are two heads better than one?. Child Development, 59, 87-96.

BEAUDICHON, J., VERBA, M. \& WINNYKAMEN, F. (1988): Interactions socilaes et acquisition de connaissances chez l'enfant: Une approche pluridimentionnelle. Revue Internationale de Psychologie Sociale, 1, 130-141.

BENSALLAH, L. (1995): Dyades asymétriques et relations amicales entre enfants. Enfance, 1, 53-69.

CAZDEN, C.B. (1988): Classroom discourse. The language of teaching and learning. London: Routledge \& Kegan Paul.

COOPER, C., MARQUIS, A., \& EDWARD, D. (1986). Four perspectives on peer learning among elementary school children. In E. Mueller \& C. Cooper (Eds.): Process and outcome in peer relationships (pp. 269-300). New York: Academic Press.

ELLIS, S. \& ROGOFF, B. (1982): The strategies and efficacy of child versus adult teacher. Child Development, 53, 730-755.

ELLIS, S. \& ROGOFF, B. (1986): Problem solving in children management of instruction. In E. C. Mueller \& C. Cooper (Eds.) Process and otucome in peer relationship, (pp. 65-92). New York: Academic Press.

FORMAN, E. \& CAZDEN, C.B. (1984): Exploring Vygotskian Perspectives in education: the cognitive value of peer interaction. En En J.V. Wertsch (comp.), Culture, communication and cognition: Vygotskian perspectives. Cambridge: Cambridge University Press, 139-157.

FRAYSSE, J.C. (1992): Relations entre développement cognitif et competénces interactives. Avalaible from The University of Provence, Aix en Prevence, France.

GILLY, M. (1980): Maître-élève. Rôles institutionnels et représentations. Paris: PUF.

GILLY, M. (1988): Interactions entre pairs et constructions cognitives: travaux expérimentaus de laboratoire au terrain pédagogique. European Journal of Psychology of Education, no Hors série"Le functionnement de l'enfant à l'école: bilans et perspectives psychologiques et didactiques", 127-138.

GILLY, M. (1989): The psychosocial mechanism of cognitive constructions. Experimental research and teaching perspectives. International Journal of Educational Rersearch, 13, 607-621.

GILLY, M. (1989b): A propos de la theorie du conglic socio-cognitif et des mecanismes psycho-sociaux des constructions: perspectives actuelles et modeles expli- 
catifs. In N. Bednarz, \& C. Garnier (Eds.) Construcions des savoirs: obstacles et conflits. Montreal: Agence d'ARC.

HARTUP, W.W. (1989): Social relationships and their developmental significance.American Psychologist, 44, 120-126.

PASTOR, E. \& SASTRE, S. (1994): Desarrollo de la inteligencia. En Bermejo, V. (Ed.): Desarrollo cognitivo. Madrid: Síntesis, pp. 191-213.

ROUX, J. P. \& GILLY, M, (1993): Social significance of task, routines, and pragmatyic schemas in distribution activities. European Journal of Social Psychology, 23, 355-371.

SANTOLINI, A., DANIS, A. \& TIJUS CH.A. (1996): Une méthode d'analyse des interactions cognitives dans l'environnement proximal du jeune enfant. Enfance, no 3/1996, pp. 331-360.

SASTRE, S. Y PASTOR, E. (en prensa): Desarrollo cognitivo y organización lógica de la acción: propuesta de análisis cualitativo en $\mathrm{M}^{\mathrm{a}} \mathrm{T}$. Anguera (Ed): Metodología observacional en la investigación Psicológica. (Vol. 5) Barcelona: PPU.

SASTRE, S. Y PASTOR, E. (1997): Desarrollo cognitivo e interacción asimétrica: notas para su estudio microgenético (en prensa).

SHUTE, R., FOOT, H., \& MORGAN, M. (1992): The sensitivity of children and adults as tutors. Educational Studies, Vol. 18, 1, 2136.

STAMBAK, M. \& SINCLAIR, H. (1993): Introduction. In M. Stambak \& Sinclair, H. (Eds.): Pretend play among 3 years old. Hillsdale NJ: Erlbaum.

VERBA, M. (1993): Cooperative Formats in Pretend Play Among Young Children. Cognition and Instruction, 11(3\&4), 265-280.

VERBA, M. (1993b): The construction and sharing of meanings in pretend play. In M. Stambak \& H. Sinclair (Eds.). Pretend Play among 3 years olds. Hillsdale, New Jersey: Lawrence Erlbaum.

VERBA, M. \& ISAMBERT, A.L. (1987): La costruzione delle conoscenze attraverso gli scambi tra bambini: Ruolo dei "Piu Vecchi" all' interno del gruppo. En A. Bondiooli \& S. Mantovani (Eds.): Manuale critico dell'asilo nido. (pp. 306-323). Milano: Franco Angeli.

VERBA, M. \& WINNYKAMEN, F. (1992): Expert-Novice Interactions: Influence of Partner Status. European Journal of Psychology of Education.

VERBA, M. (1994): The Begginins of Collaboration in Peer Interaction. Human Development, 37, 125-139.

VERBA, M (1997): Interacció $i$ desenvolupament cognitiu. Seminario celebrado en la URV (Tarragona), 16-1-1997.

VERBA. M. (1998): Tutoring between young children: how simmetry can modify asimmetrical interactions. International Journal of Behavioral Development, Volume 22, Number 1, Marck 1998, 195-216. 
VERBA, M. \& STAMBAK, M. \& SINCLAIR, H. (1982): Physical Knowledge and social interaction in children (from 18-24 months of age). In G.E. Forman (De.), Action and thought: From sensorimotor schemes to symbolic operation (pp. 267-296). New York: Academic Press.

VERBA, M. \& ISAMBERT, A.L. (1987): La costruzione delle conoscenze attraverso gli scambi tra bambini: Ruolo dei "Piu Vecchi" all' interno del gruppo. En A. Bondiooli \& S. Mantovani (Eds.): Manuale critico dell'asilo nido. (pp. 306-323). Milano: Franco Angeli.

VERBA, M. \& MARCOS, H. (1995): Les interactions éducatives à l'âge préscolaire. Research report, Contrat MIRE, December, 1995.

WERTSCH, J.V. \& HICKMANN, M. (1987): Problem solving in social interaction: A microgenetic analysis. In M. Hickman (Ed.) Social and functional approaches to language and though (pp. 251-266). Orlando, FL: Academic Press.

WOOD, D. ET MIDDLETON, D. (1975): A study of assisted problem-solving. British Journal of Psychology, 2, 181-191.

WOOD, D., BRUNER, J.S., ROSS, G. (1976): The role of tutoring in problem solving. Journal of Child Psychology and Psychiatry, 17, 89-100. 